\title{
Navier-Stokes Equations with Lower Bounds on the Pressure
}

\author{
G. SEREGIN \& V. ŠVERÁK \\ Communicated by the Editors
}

\begin{abstract}
We prove that weak solutions of the three-dimensional incompressible NavierStokes equations are smooth if the negative part of the pressure is controlled, or if the positive part of the quantity $|v|^{2}+2 p$ is controlled.
\end{abstract}

\section{Introduction}

We consider the Cauchy problem for the three-dimensional Navier-Stokes equations

$$
\partial_{t} v+\operatorname{div} v \otimes v-\Delta v+\nabla p=0, \quad \operatorname{div} v=0
$$

in $\left.\mathbb{R}^{3} \times\right] 0, \infty$, with $v(x, 0)$ smooth (or "sufficiently regular") and decaying sufficiently fast at infinity. Our main goal is to study the regularity of solutions of to the Navier-Stokes equations under certain assumptions on the pressure $p$. The pressure $p$ is a relatively well-defined quantity in real fluids. In the Navier-Stokes system, $p$ is determined only up to an arbitrary function of $t$, due to the idealized assumption of incompressibility. A way to remove this ambiguity is to specify $p$ at infinity. In the context of this work there will be no loss of generality in assuming that $p$ vanishes at infinity. (See Section 2 for a precise definition.) The pressure defined in this way will be called the normalized pressure. In what follows $p$ will always denote the normalized pressure.

Our work was motivated by the following question.

(Q) If a solution to the Navier-Stokes equations develops a singularity, must the normalized pressure become unbounded from below?

One of the main results in this work is a positive answer to this question (Theorem 2.2).

Considering a flow of water under some standard conditions, we can speculate that if $p$ becomes very low, we will encounter the phenomenon of cavitation. This 
means that in areas of very low pressure bubbles of water vapor will form in the fluid. Since the areas of very low pressure must have small volume, we can expect that eventually the bubbles will be carried into an area where the pressure is not so low, and will collapse. The collapse of even very small bubbles should create observable effects (e.g., popping sounds). As far as we know, cavitation is not observed in reasonable flows, such as flows in pipes under normal temperature and pressure, even when the Reynolds number is high. Therefore, for such flows we can assume that $p$ does not become exceedingly low. Hence, by the result above, $v$ should be smooth. We can further speculate that this means that all singularities of solutions to the Navier-Stokes must be unstable, if they exist at all. This was conjectured in [18].

We prove a slightly stronger statement than suggested by $(\mathrm{Q})$, in that we do not need a point-wise condition $p(x, t) \geqq-C$ to get regularity, but only a weaker integral condition is necessary (see (2.7) and (2.8)).

It turns out that our method also gives a proof of the following statement, which is of independent interest: If the quantity $|v|^{2}+2 p$ is bounded from above, the solution must be regular. This is related to the works [7] and [26] on the fivedimensional steady-state Navier-Stokes equations.

We briefly outline the main idea of the proof. The key is the following identity:

$$
\begin{aligned}
\int_{B\left(x_{0}, R\right)} & \frac{1}{\left|y-x_{0}\right|}\left(2 p(y, t)+\left|\widehat{v}^{x_{0}}(y, t)\right|^{2}\right) d y \\
& =\frac{1}{R} \int_{B\left(x_{0}, R\right)}\left(3 p(y, t)+|v(y, t)|^{2}\right) d y \\
& =R^{2} \int_{\mathbb{R}^{3}-B\left(x_{0}, R\right)} \nabla_{y}^{2}\left(\frac{1}{\left|y-x_{0}\right|}\right):(v(y, t) \otimes v(y, t)) d y,
\end{aligned}
$$

where $\hat{v}^{x_{0}}(x, t)$ is the orthogonal projection of $v(x, t)$ into the two-dimensional subspace of $\mathbb{R}^{3}$ perpendicular to $x-x_{0}$. We can see that bounds for the negative part of $p$ or the positive part of $|v|^{2}+2 p$ give non-trivial estimates for $v$. A key feature of these estimates is that the controlled quantities are invariant under the natural scaling of the equation $v(x, t) \rightarrow \lambda v\left(\lambda x, \lambda^{2} t\right)$. In the language of regularity theory, under our assumptions the above identity gives estimates which move the equation from the realm of "super-critical" to the realm of "critical". This makes the problem manageable.

Other papers where regularity for weak solutions to the Navier-Stokes equations is studied under various assumptions on pressure include $[1-3,5,20]$. Regularity criteria involving other quantities can be found for example in $[22,23,17,12,8,25$, $6]$. 


\section{Notation and main results}

We denote by $\mathbb{M}^{3}$ the space of all real $3 \times 3$ matrices. Adopting summation over repeated Latin indices, running from 1 to 3 , we shall use the following notation:

$$
\begin{gathered}
u \cdot v=u_{i} v_{i}, \quad|u|=\sqrt{u \cdot u}, \quad u=\left(u_{i}\right) \in \mathbb{R}^{3}, \quad v=\left(v_{i}\right) \in \mathbb{R}^{3} ; \\
A: B=\operatorname{tr} A^{*} B=A_{i j} B_{i j}, \quad|A|=\sqrt{A: A}, \\
A^{*}=\left(A_{j i}\right), \quad \operatorname{tr} A=A_{i i}, \quad A=\left(A_{i j}\right) \in \mathbb{M}^{3}, \quad B=\left(B_{i j}\right) \in \mathbb{M}^{3} ; \\
u \otimes v=\left(u_{i} v_{j}\right) \in \mathbb{M}^{3}, \quad A u=\left(A_{i j} u_{j}\right) \in \mathbb{R}^{3}, \quad u, v \in \mathbb{R}^{3}, \quad A \in \mathbb{M}^{3} .
\end{gathered}
$$

Let $\omega$ be a domain in some finite-dimensional space. We denote by $L_{m}\left(\omega ; \mathbb{R}^{l}\right)$ and $W_{m}^{1}\left(\omega ; \mathbb{R}^{l}\right)$ the known Lebesgue and Sobolev spaces of functions from $\omega$ into $\mathbb{R}^{l}$. The norm of the space $L_{m}\left(\omega ; \mathbb{R}^{l}\right)$ is denoted by $\|\cdot\|_{m, \omega}$. If $m=2$, then we use the abbreviation $\|\cdot\|_{\omega} \equiv\|\cdot\|_{2, \omega}$.

Let $T$ be a positive parameter, $\Omega$ be a domain in $\mathbb{R}^{3}$. We denote by $Q_{T} \equiv$ $\Omega \times] 0, T$ [ the space-time cylinder. Space-time points are denoted by $z=(x, t)$, $z_{0}=\left(x_{0}, t_{0}\right)$, etc. Let $L_{m, n}\left(Q_{T} ; \mathbb{R}^{l}\right)$ be the space of measurable $\mathbb{R}^{l}$-valued functions with the following norm:

$$
\|f\|_{m, n, Q_{T}}= \begin{cases}\left(\int_{0}^{T}\|f(\cdot, t)\|_{m, \Omega}^{n} d t\right)^{\frac{1}{n}}, & n \in[1,+\infty[, \\ \operatorname{ess} \sup _{t \in[0, T]}\|f(\cdot, t)\|_{m, \Omega}, & n=+\infty .\end{cases}
$$

In the special case $\Omega=\mathbb{R}^{3}$ and $T=+\infty$, we use the abbreviations

$$
\begin{gathered}
L_{m}\left(\Omega ; \mathbb{R}^{3}\right)=L_{m}, \quad W_{2}^{1}\left(\Omega ; \mathbb{R}^{3}\right)=H^{1}, \quad L_{m, n}\left(Q_{T} ; \mathbb{R}^{3}\right)=L_{m, n}, \\
L_{m}\left(0, T ; W_{2}^{1}\left(\Omega ; \mathbb{R}^{3}\right)\right)=L_{m}\left(H^{1}\right) .
\end{gathered}
$$

For integrable-in- $Q_{T}$ scalar-valued, vector-valued, and tensor-valued functions, we shall use the following differential operators

$$
\begin{gathered}
\partial_{t} v=\frac{\partial v}{\partial t}, \quad v_{, i}=\frac{\partial v}{\partial x_{i}}, \quad \nabla p=\left(p_{, i}\right), \quad \nabla u=\left(u_{i, j}\right), \\
\operatorname{div} v=v_{i, i}, \quad \operatorname{div} \tau=\left(\tau_{i j, j}\right), \quad \Delta u=\operatorname{div} \nabla u,
\end{gathered}
$$

which are understood in the sense of distributions. Here $x_{i}, i=1,2,3$, are the Cartesian coordinates of a point $x \in \mathbb{R}^{3}$, and $\left.t \in\right] 0, T[$ is the time variable.

For balls and parabolic cylinders, we use the standard notation:

$$
\left.B\left(x_{0}, R\right) \equiv\left\{x \in \mathbb{R}^{3} \quad \| \quad\left|x-x_{0}\right|<R\right\}, \quad Q\left(z_{0}, R\right) \equiv B\left(x_{0}, R\right) \times\right] t_{0}-R^{2}, t_{0}[,
$$

where $z_{0}=\left(x_{0}, t_{0}\right)$. 
Let us formulate the main results of the paper. To this end, we remind the reader that for the initial data satisfying the conditions

$$
v_{0} \in H^{1}, \quad \operatorname{div} v_{0}=0 \quad \text { in } \mathbb{R}^{3},
$$

the Cauchy problem

$$
\left.\left.\begin{array}{rl}
\partial_{t} v+\operatorname{div}(v \otimes v)-\Delta v+\nabla p & =0 \\
\operatorname{div} v & =0
\end{array}\right\} \text { in } \mathbb{R}^{3} \times\right] 0,+\infty[,
$$

always has a so-called Leray-Hopf weak solution (see [15, 10,11,13]). This means that there exists at least one function $v$ with the following properties:

$$
v \in L_{2, \infty} \cap L_{2}\left(H^{1}\right), \quad \operatorname{div} v(\cdot, t)=0 \quad \text { in } \quad \mathbb{R}^{3} \quad \text { for all } t \geqq 0 ;
$$$$
\text { the function } t \mapsto \int_{\mathbb{R}^{3}} v(\cdot, t) \cdot w(\cdot) d x \quad \text { is continuous on } \quad[0,+\infty[
$$$$
\text { for all } w \in L_{2} \text {; }
$$

$$
\int_{\left.\mathbb{R}^{3} \times\right] 0, \infty[}\left\{-v \cdot \partial_{t} w-v \otimes v: \nabla w+\nabla v: \nabla w\right\} d x=0
$$

for any $w \in C_{0}^{\infty}\left(\mathbb{R}^{3} \times\right] 0, \infty\left[; \mathbb{R}^{3}\right)$ such that $\operatorname{div} w(\cdot, t)=0$ for all $t>0$;

$$
\begin{gathered}
\left\|v(\cdot, t)-v_{0}(\cdot)\right\|_{\mathbb{R}^{3}} \rightarrow 0 \quad \text { as } \quad t \rightarrow 0+0 ; \\
\int_{\mathbb{R}^{3}}|v(x, t)|^{2} d x+2 \int_{0}^{t} \int_{\mathbb{R}^{3}}\left|\nabla v\left(x, t^{\prime}\right)\right|^{2} d x d t^{\prime} \leqq \int_{\mathbb{R}^{3}}\left|v_{0}(x)\right|^{2} d x \\
\text { for all } t \geqq 0 .
\end{gathered}
$$

In this formulation, no information about the pressure $p$ is given.

However, using the uniqueness theorem and the coercive $L_{s, l}$ estimates of solutions to the Cauchy problem for the Stokes equations (see, for example, $[9,19]$, and $[11,13,24]$ in the case $s=l$ ), pressure can be introduced in a natural way. More precisely, it can be proved (see, for instance [4] and [14]) that there exists a function $p \in L_{1, \text { loc }}$ such that, for $O<\delta<T<+\infty$,

$$
\nabla p \in L_{s, l}\left(\mathbb{R}^{3} \times\right] \delta, T\left[; \mathbb{R}^{3}\right),
$$

where

$$
\frac{3}{s}+\frac{2}{l} \geqq 4
$$


Moreover,

$$
\partial_{t} v \in L_{s, l}\left(\mathbb{R}^{3} \times\right] \delta, T\left[; \mathbb{R}^{3}\right), \quad \nabla^{2} v \in L_{s, l}\left(\mathbb{R}^{3} \times\right] \delta, T\left[; \mathbb{M}^{3} \times \mathbb{R}^{3}\right),
$$

and the equation

$$
\partial_{t} v+\operatorname{div}(v \otimes v)-\Delta v+\nabla p=0
$$

holds a.e. in $\left.\mathbb{R}^{3} \times\right] 0, \infty[$.

The pressure $p$ is determined up to an arbitrary function of $t$. We fix a representative for $p$ by setting

$$
p(x, t) \equiv \frac{1}{4 \pi} \int_{\mathbb{R}^{3}} \frac{1}{|x-y|} \operatorname{div} \operatorname{div}(v(y, t) \otimes v(y, t)) d y .
$$

To show that this function satisfies (2.5), let us denote the function on the right-hand side of (2.6) by $p_{0}$. It is known that

$$
\Delta p_{0}(x, t)=-\operatorname{div} \operatorname{div}(v(x, t) \otimes v(x, t)) .
$$

Differentiation in $x$ gives us:

$$
\nabla p_{0}=\frac{1}{3} G+T(G)
$$

where $G \equiv v_{k} v_{, k}=\left(v_{k} v_{i, k}\right)$ and

$$
\begin{aligned}
T(G)(x, t) & \equiv-\frac{1}{4 \pi} \int_{\mathbb{R}^{3}} \nabla_{x}^{2}\left(\frac{1}{|x-y|}\right) G(y, t) d y \\
& =-\frac{1}{4 \pi} \int_{\mathbb{R}^{3}}\left(\left(\frac{\delta_{i j}}{|x-y|^{3}}-\frac{3\left(x_{i}-y_{i}\right)\left(x_{j}-y_{j}\right)}{|x-y|^{5}}\right) G_{j}(y, t)\right) d y
\end{aligned}
$$

is a singular integral. According to the boundedness of singular integrals in $L_{s}$, we have the estimate

$$
\int_{\mathbb{R}^{3}}\left|\nabla p_{0}(x, t)\right|^{\frac{9}{8}} d x \leqq c_{1} \int_{\mathbb{R}^{3}}(|v(x, t)||\nabla v(x, t)|)^{\frac{9}{8}} d x
$$

for all positive $t$ and for some absolute constant $c_{1}$.

Next, by Hölder's inequality and by the multiplicative inequality, we obtain

$$
\begin{aligned}
\int_{\mathbb{R}^{3}}|v|^{\frac{9}{8}}|\nabla v|^{\frac{9}{8}} d x & \leqq\left(\int_{\mathbb{R}^{3}}|\nabla v|^{2} d x\right)^{\frac{9}{16}}\left(\int_{\mathbb{R}^{3}}|v|^{\frac{18}{7}} d x\right)^{\frac{7}{18}} \\
& \leqq c_{2}\left(\int_{\mathbb{R}^{3}}|\nabla v|^{2} d x\right)^{\frac{3}{4}}\left(\int_{\mathbb{R}^{3}}|v|^{2} d x\right)^{\frac{3}{2}} .
\end{aligned}
$$


Thus,

$$
\nabla p_{0}(\cdot, t) \in L_{\frac{9}{8}}
$$

for a.e. $t>0$.

On the other hand, it follows from equation (2.5) that

$$
\Delta p(x, t)=-\operatorname{div} \operatorname{div}(v(x, t) \otimes v(x, t)) .
$$

Therefore, $q \equiv p-p_{0}$ is a harmonic function in $x$ for a.e. $t>0$. But, by (2.4),

$$
\nabla p \in L_{\frac{9}{8}, \frac{3}{2}}\left(\mathbb{R}^{3} \times\right] \delta, T\left[; \mathbb{R}^{3}\right) .
$$

This means that

$$
\nabla q(\cdot, t) \in L_{\frac{9}{8}}\left(\mathbb{R}^{3} \times\right] \delta, T\left[; \mathbb{R}^{3}\right)
$$

for a.e. $t>0$. Since $q$ is harmonic in $x$, we find that $\nabla q(\cdot, t)=0$ in $\mathbb{R}^{3}$ and, therefore, $q$ is a function of $t$ only.

Definition 2.1. We say that a function $\left.g: \mathbb{R}^{3} \times\right] 0,+\infty[\rightarrow[0,+\infty[$ satisfies condition (C) if, for any $t_{0}>0$, there exists a positive number $R_{0}=R_{0}\left(t_{0}\right)$ such that

$$
A\left(t_{0}\right) \equiv \sup _{x_{0} \in \mathbb{R}^{3}} \sup _{t_{0}-R_{0}^{2} \leqq t \leqq t_{0}} \int_{B\left(x_{0}, R_{0}\right)} \frac{g(x, t)}{\left|x-x_{0}\right|} d x<+\infty
$$

and,

$$
\begin{aligned}
& \text { for each fixed } \left.\left.x_{0} \in \mathbb{R}^{3} \text { and for each fixed } R \in\right] 0, R_{0}\right], \\
& \text { the function } t \mapsto \int_{B\left(x_{0}, R\right)} \frac{g(x, t)}{\left|x-x_{0}\right|} d x \text { is continuous at } t_{0} \text { from the left. }
\end{aligned}
$$

Our main result is as follows

Theorem 2.2. Let $v$ be a Leray-Hopf solution to the Cauchy problem (2.1)-(2.3) and let $p$ be the normalized pressure associated with $v$. Assume that there exists a function $g$ satisfying condition (C) such that

$$
\left.|v(x, t)|^{2}+2 p(x, t) \leqq g(x, t), \quad x \in \mathbb{R}^{3}, t \in\right] 0,+\infty[
$$

or

$$
\left.p(x, t) \geqq-g(x, t), \quad x \in \mathbb{R}^{3}, t \in\right] 0,+\infty[.
$$

Then $v$ is Hölder continuous on $\left.\mathbb{R}^{3} \times\right] 0,+\infty[$ and therefore smooth and unique.

Remark 2.3. Obviously, conditions (2.7) and (2.8) are satisfied if $g \equiv$ constant $>0$ in $\left.\mathbb{R}^{3} \times\right] 0,+\infty[$. 


\section{Remarks on suitable weak solutions to the Navier-Stokes equations}

In this section, we are going to discuss some facts about the so-called suitable weak solutions to the Navier-Stokes equations:

$$
\left.\begin{array}{rl}
\partial_{t} v+\operatorname{div} v \otimes v-\Delta v & =f-\nabla p \\
\operatorname{div} v & =0
\end{array}\right\} \quad \text { in } \quad Q_{T},
$$

where $\left.Q_{T} \equiv \Omega \times\right] 0, T\left[, \Omega\right.$ is a domain in $\mathbb{R}^{3}$, and $T$ is a positive parameter. We always assume that $f$ lies in the Morrey space

$$
M_{2, \gamma}\left(Q_{T} ; \mathbb{R}^{3}\right) \equiv\left\{f \in L_{2}\left(Q_{T} ; \mathbb{R}^{3}\right) \| d_{\gamma}\left(f ; Q_{T}\right)<+\infty\right\}
$$

for some positive number $\gamma$, where

$$
d_{\gamma}\left(f ; Q_{T}\right) \equiv \sup \left\{\frac{1}{R^{\gamma+1 / 2}}\left(\int_{Q\left(x_{0}, R\right)}|f|^{2} d x\right)^{\frac{1}{2}} \| Q\left(x_{0}, R\right) \Subset Q_{T}, R>0\right\} .
$$

We say that a pair of functions $v$ and $p$ are suitable weak solutions of the NavierStokes equations if the following conditions hold (see $[21,4,16,14]$ for details):

$$
v \in L_{2, \infty}\left(Q_{T} ; \mathbb{R}^{3}\right) \cap L_{2}\left(0, T ; W_{2}^{1}\left(\Omega ; \mathbb{R}^{3}\right)\right), \quad p \in L_{\frac{3}{2}}\left(Q_{T}\right) ;
$$

equations (3.1) are satisfied in $Q_{T}$ in the sense of distributions;

and

$$
\begin{aligned}
& \int_{\Omega}|v(x, t)|^{2} \phi(x, t) d x+2 \int_{0}^{t} \int_{\Omega}\left|\nabla v\left(x, t^{\prime}\right)\right|^{2} \phi\left(x, t^{\prime}\right) d x d t^{\prime} \\
& \leqq \\
& \quad \int_{0}^{t} \int_{\Omega}\left\{\left|v\left(x, t^{\prime}\right)\right|^{2}\left(\partial_{t} \phi\left(x, t^{\prime}\right)+\Delta \phi\left(x, t^{\prime}\right)\right)\right. \\
& \quad+2 f\left(x, t^{\prime}\right) \cdot v\left(x, t^{\prime}\right) \phi\left(x, t^{\prime}\right) \\
& \left.\quad+\left(\left|v\left(x, t^{\prime}\right)\right|^{2}+2 p\left(x, t^{\prime}\right)\right) v\left(x, t^{\prime}\right) \cdot \nabla \phi\left(x, t^{\prime}\right)\right\} d x d t^{\prime}
\end{aligned}
$$

for a.e. $t \in] 0, T$ [ and for all non-negative functions $\phi \in C_{0}^{\infty}\left(\mathbb{R}^{3} \times \mathbb{R}\right)$ vanishing in a neighborhood of the parabolic boundary $\partial^{\prime} Q_{T} \equiv \Omega \times\{t=0\} \cup \partial \Omega \times[0, T]$.

As in [14], we call a point $z_{0} \in Q_{T}$ regular for $v$ if there exists a non-empty neighborhood $\mathcal{O}_{z_{0}}$ of this point where the function $z \mapsto v(z)$ has a Hölder continuous representative. It can be proved that there exists a representative of $v$ such that (see $[21,4,16,14]$ for details)

$$
\mathcal{H}^{1}(\Sigma)=0,
$$


where $\Sigma$ is the set of all singular points of $v$ and $\mathcal{H}^{1}$ is the one-dimensional parabolic Hausdorff measure. By definition,

$$
\mathcal{H}^{1}(\Sigma) \equiv \lim _{\delta \rightarrow 0} \inf \left\{\sum_{i} R_{i} \| \Sigma \subset \sum_{i} Q\left(z_{i}, R_{i}\right), 0<R_{i} \leqq \delta\right\} .
$$

In what follows, we shall fix a representative of $v$ such that

$$
\liminf _{t \rightarrow t_{0}} \int_{\Omega}|v(x, t)|^{2} d x \geqq \int_{\Omega}\left|v\left(x, t_{0}\right)\right|^{2} d x \quad \text { for all } 0<t_{0}<T
$$

and, for each $w \in L_{2}\left(\Omega, \mathbb{R}^{3}\right)$,

$$
t \in] 0, T\left[\mapsto \int_{\Omega} v(x, t) \cdot w(x) d x\right. \text { is a continuous function. }
$$

To see that this is possible we note that, by (3.5),

$$
\mathcal{H}^{1}\left(\Omega \times\left\{t=t_{0}\right\} \cap \Sigma\right)=0
$$

and, according to the definition of regular points,

$$
v(x, t) \rightarrow v\left(x, t_{0}\right) \quad \text { for a.e. } x \in \Omega .
$$

Therefore (3.6) follows from Fatou's lemma. On the other hand, by (3.2), we have

$$
\left\|v\left(\cdot, t_{0}\right)\right\|_{2, \Omega} \leqq\|v\|_{2, \infty, Q_{T}}
$$

for all $\left.t_{0} \in\right] 0, T[$, and thus, by (3.8) and (3.9),

$$
v(\cdot, t) \rightarrow v\left(\cdot, t_{0}\right) \quad \text { in } \quad L_{r}\left(\Omega ; \mathbb{R}^{3}\right)
$$

for any $r \in[1,2[$. In turn, (3.9) and (3.10) imply (3.7).

Remark 3.1. Following the arguments in [14], we can see that all the above statements remain valid for $t_{0}=T$.

Lemma 3.2. Let $v$ be as above. Given $\Omega_{0} \Subset \Omega, 0<t_{0} \leqq T$, and $0<\delta_{0}<\sqrt{t_{0}}$, assume that

$$
\begin{array}{r}
a\left(\Omega_{0}, t_{0}, \delta_{0}\right) \equiv \sup \left\{\frac{1}{R} \int_{B\left(x_{0}, R\right)}|v(x, t)|^{2} d x \quad \| x_{0} \in \Omega_{0},\right. \\
\left.t \in\left[t_{0}-\delta_{0}^{2}, t_{0}\right], \quad 0<R \leqq d_{0} \equiv \frac{1}{2} \operatorname{dist}\left(\partial \Omega, \Omega_{0}\right)\right\} \\
<+\infty .
\end{array}
$$

Then,

$$
\lim _{t \rightarrow t_{0}-0} \int_{\Omega_{0}}\left|v(x, t)-v\left(x, t_{0}\right)\right|^{2} d x=0
$$


Proof. Taking into account (3.7), we see that it is enough to prove

$$
\lim _{t \rightarrow t_{0}-0} \int_{\Omega_{0}}|v(x, t)|^{2} d x=\int_{\Omega_{0}}\left|v\left(x, t_{0}\right)\right|^{2} d x .
$$

We first note that (3.5) implies the following fact. For each $\gamma$, there exists a countable family of sets of the form

$$
b_{i}^{\gamma, t_{0}} \equiv B\left(x_{i}^{\gamma}, R_{\gamma i}\right) \times\left\{t=t_{0}\right\}
$$

such that

$$
R_{\gamma i} \leqq d_{0}, \quad \Sigma \cap\left(\bar{\Omega}_{0} \times\left\{t=t_{0}\right\}\right) \subset \sum_{i} b_{i}^{\gamma, t_{0}}, \quad \sum_{i} R_{\gamma i}<\gamma
$$

Let us fix $\varepsilon>0$ and let

$$
\gamma=\frac{\varepsilon}{8 a\left(\Omega_{0}, t_{0}, \delta_{0}\right)} .
$$

Then, by (3.11) and (3.14), we obtain

$$
\begin{aligned}
& \left.\left|\int_{\sum_{i} B\left(x_{i}^{\gamma}, R_{\gamma i}\right)}\right| v(x, t)\right|^{2} d x-\int_{\sum_{i} B\left(x_{i}^{\gamma}, R_{\gamma i}\right)}\left|v\left(x, t_{0}\right)\right|^{2} d x \mid \\
& \quad \leqq \sum_{i} \int_{B\left(x_{i}^{\gamma}, R_{\gamma i}\right)}|v(x, t)|^{2} d x+\sum_{i} \int_{B\left(x_{i}^{\gamma}, R_{\gamma i}\right)}\left|v\left(x, t_{0}\right)\right|^{2} d x \\
& \quad \leqq 2 a\left(\Omega_{0}, t_{0}, \delta_{0}\right) \sum_{i} R_{\gamma i}<2 \gamma a\left(\Omega_{0}, t_{0}, \delta_{0}\right) \\
& \quad \leqq \frac{\varepsilon}{4}
\end{aligned}
$$

for all $t \in\left[t_{0}-\delta_{0}^{2}, t_{0}\right]$.

We let

$$
\omega^{\gamma} \equiv \bar{\Omega}_{0} \times\left\{t=t_{0}\right\}-\sum_{i} b_{i}^{\gamma, t_{0}} .
$$

For each $z \in \omega^{\gamma}$, there exists a non-empty neighborhood $\mathcal{O}_{z}$ such that the function $z \mapsto v(z)$ is Hölder continuous on $\mathcal{O}_{z} \cap \bar{Q}_{T}$. Since $\omega^{\gamma}$ is compact, there exists a non-empty neighborhood $\mathcal{O}_{\omega}^{\gamma}$ of the set $\omega^{\gamma}$ such that

$$
\omega^{\gamma} \subset \mathcal{O}_{\omega}^{\gamma}
$$

and the function $z \rightarrow v(z)$ is continuous in $\overline{\mathcal{O}}_{\omega}^{\gamma} \cap \bar{Q}_{T}$. Hence,

$$
\left.\left|\int_{\omega^{\gamma}}\right| v(x, t)\right|^{2} d x-\int_{\omega^{\gamma}}\left|v\left(x, t_{0}\right)\right|^{2} d x \mid<\frac{\varepsilon}{2}
$$


for all $0 \leqq t_{0}-t<\mu=\mu\left(\varepsilon, \Omega_{0}, t_{0}, \delta_{0}\right) \leqq \delta_{0}^{2}$. Combining (3.15) and (3.16), we obtain

$$
\begin{aligned}
\left.\left|\int_{\Omega_{0}}\right| v(x, t)\right|^{2} d x-\int_{\Omega_{0}}\left|v\left(x, t_{0}\right)\right|^{2} d x \mid \leqq & \left.\left|\int_{\omega^{\gamma}}\right| v(x, t)\right|^{2} d x-\int_{\omega^{\gamma}}\left|v\left(x, t_{0}\right)\right|^{2} d x \mid \\
& +\left.\left|\int_{\sum_{i} B\left(x_{i}^{\gamma}, R_{\gamma i}\right)}\right| v(x, t)\right|^{2} d x \\
& -\int_{\sum_{i} B\left(x_{i}^{\gamma}, R_{\gamma i}\right)}\left|v\left(x, t_{0}\right)\right|^{2} d x \mid \\
<\varepsilon &
\end{aligned}
$$

for all $0 \leqq t_{0}-t<\mu$. Therefore (3.13) and Lemma (3.2) are proved.

In what follows, we are going to use the following condition for local Hölder continuity of $v$.

Lemma 3.3. Let a pair of $v$ and $p$ be an arbitrary suitable weak solution to the Navier-Stokes equations in $Q_{T}$ with external force $f \in M_{2, \gamma}\left(Q_{T} ; \mathbb{R}^{3}\right)$ for some positive number $\gamma$. There exists a positive number $\varepsilon_{\star}$, depending on $\gamma$ only and having the following property. Assume that, for some positive $R_{\star}, Q\left(z_{0}, R_{\star}\right) \subset Q_{T}$ and

$$
\sup _{0<R<R_{\star}} A\left(z_{0}, R\right)<\varepsilon_{\star}
$$

where

$$
A\left(z_{0}, R\right) \equiv \sup _{t_{0}-R^{2} \leqq t \leqq t_{0}} \frac{1}{R} \int_{B\left(x_{0}, R\right)}|v(x, t)|^{2} d x .
$$

Then, $z_{0}$ is a regular point of $v$.

Proof. Our proof is mostly based on the method developed by LIN in [16] (see also [14]). As in [14], we introduce the following functionals:

$$
\begin{array}{ll}
A(\rho) \equiv A\left(z_{0}, \rho\right), & E(\rho) \equiv \frac{1}{r} \int_{Q\left(z_{0}, \rho\right)}|\nabla v|^{2} d z, \\
C(r) \equiv \frac{1}{r^{2}} \int_{Q\left(z_{0}, \rho\right)}|v|^{3} d z, & D(r) \equiv \frac{1}{r^{2}} \int_{Q\left(z_{0}, \rho\right)}|p|^{\frac{3}{2}} d z .
\end{array}
$$

We have assumed that $Q\left(z_{0}, \rho\right) \subset Q_{T}$.

In [14], the following decay estimates involving the above functionals are proved:

$$
C(r) \leqq c_{1}\left[\left(\frac{r}{\rho}\right)^{3} A^{\frac{3}{2}}(\rho)+\left(\frac{\rho}{r}\right)^{3} A^{\frac{3}{4}}(\rho) E^{\frac{3}{4}}(\rho)\right]
$$


for all $0<r \leqq \rho($ see Lemma 5.1 in [14]),

$$
A\left(\frac{\rho}{2}\right)+E\left(\frac{\rho}{2}\right) \leqq c_{1}\left[C^{\frac{2}{3}}(\rho)+C^{\frac{1}{3}}(\rho) D^{\frac{2}{3}}(\rho)+C(\rho)+d_{\gamma}^{2} \rho^{2(\gamma+1)}\right]
$$

(see inequality (5.4) in [14]),

$$
D(r) \leqq c_{1}\left[\frac{r}{\rho} D(\rho)+\left(\frac{\rho}{r}\right)^{2}\left(A^{\frac{3}{4}}(\rho) E^{\frac{3}{4}}(\rho)+d_{\gamma}^{\frac{3}{2}} \rho^{\frac{3}{2}(\gamma+1)}\right)\right]
$$

for all $r \in] 0, \rho]$ (see Lemma 5.3 in [14]). Here $d_{\gamma} \equiv d_{\gamma}\left(f ; Q_{T}\right)$ and $c_{1}$ is an absolute positive constant.

In contrast to [14], we focus on the functional

$$
\mathcal{F}(R) \equiv C(R)+D(R) .
$$

Let $\theta \in] 0,1 / 2\left[\right.$ and $Q\left(z_{0}, \rho\right) \subset Q_{T}$. We shall fix numbers $\theta$ and $\rho$ later. From Young's inequality and from (3.19), we can derive

$$
A\left(\frac{\rho}{2}\right)+E\left(\frac{\rho}{2}\right) \leqq c_{2}\left[\mathcal{F}^{\frac{2}{3}}(\rho)+\mathcal{F}(\rho)+d_{\gamma}^{2} \rho^{2(\gamma+1)}\right]
$$

where $c_{2}$ is an absolute constant. Combining estimates (3.18) and (3.21), we obtain

$$
\begin{aligned}
C(r) \leqq & c_{1}\left[\left(\frac{2 r}{\rho}\right)^{3} A^{\frac{3}{2}}\left(\frac{\rho}{2}\right)+\left(\frac{\rho}{2 r}\right)^{3} A^{\frac{3}{4}}\left(\frac{\rho}{2}\right) E^{\frac{3}{4}}\left(\frac{\rho}{2}\right)\right] \\
\leqq & c_{3}\left[\left(\frac{r}{\rho}\right)^{3} A^{\frac{3}{2}}(\rho)\right. \\
& \left.+\left(\frac{\rho}{r}\right)^{3} A^{\frac{3}{4}}(\rho)\left(\mathcal{F}^{\frac{2}{3}}(\rho)+\mathcal{F}(\rho)+d_{\gamma}^{2} \rho^{2(\gamma+1)}\right)^{\frac{3}{4}}\right]
\end{aligned}
$$

for all $0<r \leqq \rho / 2$, with $c_{3}$ an absolute constant. The same can be done with estimate (3.20). As a result, we have

$$
\begin{aligned}
D(r) \leqq & c_{4}\left[\frac{r}{\rho} \mathcal{F}(\rho)+\left(\frac{\rho}{r}\right)^{2}\left(A^{\frac{3}{4}}(\rho)\left(\mathcal{F}^{\frac{2}{3}}(\rho)+\mathcal{F}(\rho)+d_{\gamma}^{2} \rho^{2(\gamma+1)}\right)^{\frac{3}{4}}\right.\right. \\
& \left.\left.+d_{\gamma}^{\frac{3}{2}} \rho^{\frac{3}{2}(\gamma+1)}\right)\right],
\end{aligned}
$$

for all $0<r \leqq \rho / 2$, where $c_{4}$ is an absolute constant.

Setting $\theta=r / \rho$, we observe that from (3.22) and (3.23) we can obtain the following estimate:

$$
\begin{aligned}
\mathcal{F}(\theta \rho) \leqq & c_{5}\left[\theta \mathcal{F}(\rho)+\theta^{3} A^{\frac{3}{2}}(\rho)+d_{\gamma}^{\frac{3}{2}} \rho^{\frac{3}{2}(\gamma+1)}\right. \\
& \left.+\left(\theta^{-3}+\theta^{-2}\right) A^{\frac{3}{4}}(\rho)\left(\mathcal{F}^{\frac{2}{3}}(\rho)+\mathcal{F}(\rho)+d_{\gamma}^{2} \rho^{2(\gamma+1)}\right)^{\frac{3}{4}}\right] \\
\leqq & c_{6}\left[\theta \mathcal{F}(\rho)+\frac{1}{\theta^{15}}\left(A^{3}(\rho)+A^{\frac{3}{2}}(\rho)+A^{\frac{3}{4}}(\rho) d_{\gamma}^{\frac{3}{2}} \rho^{\frac{3}{2}(\gamma+1)}\right)\right. \\
& \left.+d_{\gamma}^{\frac{3}{2}} \rho^{\frac{3}{2}(\gamma+1)}\right] .
\end{aligned}
$$


Here, $c_{5}$ and $c_{6}$ are absolute constants and $\left.\theta \in\right] 0,1 / 2[$.

Let us fix $\theta \in] 0,1 / 2\left[\right.$ and $\left.\left.\rho_{0} \in\right] 0, R_{\star}\right]$ in such a way that

$$
\theta c_{6} \leqq \frac{1}{2}, \quad d_{\gamma}^{\frac{3}{2}} \rho_{0}^{\frac{3}{2}(\gamma+1)} \leqq \varepsilon_{\star} .
$$

Without loss of generality, we may assume that $\varepsilon_{\star} \leqq 1$. Then, (3.24) and (3.25) imply the bound

$$
\mathcal{F}(\theta \rho) \leqq \frac{1}{2} \mathcal{F}(\rho)+c_{7} \varepsilon_{\star},
$$

for any $\rho \in] 0, \rho_{0}$ ], with $c_{7}$ an absolute constant. Iterating (3.26), we obtain

$$
\mathcal{F}\left(\frac{\rho}{2^{k}}\right) \leqq \frac{1}{2^{k}} \mathcal{F}(\rho)+2 c_{7} \varepsilon_{\star}
$$

for all natural $k$. The last estimate implies

$$
\liminf _{R \rightarrow 0+0} \mathcal{F}(R) \leqq 2 c_{7} \varepsilon_{\star}
$$

According to Proposition 2.8 in [14], there exists $\bar{\varepsilon}_{0}(\gamma)$ such that if

$$
\liminf _{R \rightarrow 0+0}\left\{\left(\frac{3}{4 \pi} C(R)\right)^{\frac{1}{3}}+\left(\frac{3}{4 \pi} D(R)\right)^{\frac{2}{3}}\right\}<\bar{\varepsilon}_{0}(\gamma),
$$

then $z_{0}$ is regular point. Choosing $\varepsilon_{\star}$ in an appropriate way, we deduce the statement of the lemma from (3.27) and (3.28). Lemma 3.3 is proved.

\section{Proof of Theorem 2.2}

First, let us prove that, for functions $v$ and $p$ connected by relation (2.6), and for any $x_{0} \in \mathbb{R}^{3}$, for any $t>0$, and for any $R>0$, the following identities are valid:

$$
\begin{aligned}
\int_{B\left(x_{0}, R\right)} & \frac{1}{\left|y-x_{0}\right|}\left(2 p(y, t)+\left|\widehat{v}^{x_{0}}(y, t)\right|^{2}\right) d y \\
& =\int_{B\left(x_{0}, R\right)} \frac{1}{R}\left(3 p(y, t)+|v(y, t)|^{2}\right) d y \\
& =R^{2} \int_{\mathbb{R}^{3}-B\left(x_{0}, R\right)} \nabla_{y}^{2}\left(\frac{1}{\left|y-x_{0}\right|}\right):(v(y, t) \otimes v(y, t)) d y,
\end{aligned}
$$

where

$$
\widehat{v}^{x_{0}}(y, t) \equiv v(y, t)-\widetilde{v}^{x_{0}}(y, t), \quad \widetilde{v}^{x_{0}}(y, t) \equiv \frac{v(y, t) \cdot\left(y-x_{0}\right)\left(y-x_{0}\right)}{\left|y-x_{0}\right|^{2}} .
$$


To this end, we take a sufficiently regular function $g:] 0,+\infty[\rightarrow[0,+\infty[$ and observe that, by (2.6),

$$
\begin{aligned}
\int_{B\left(x_{0}, R\right)} & g\left(\left|x_{0}-y\right|\right) p(y, t) d y \\
= & \frac{1}{4 \pi} \int_{\mathbb{R}^{3}} f(x, t) \int_{B\left(x_{0}, R\right)} g\left(\left|x_{0}-y\right|\right) \frac{1}{|x-y|} d y d x,
\end{aligned}
$$

where $f(x, t) \equiv \operatorname{div} \operatorname{div}(v(x, t) \otimes v(x, t))$. It is easy to check that

$$
\begin{aligned}
& \int_{B\left(x_{0}, R\right)} g\left(\left|x_{0}-y\right|\right) \frac{1}{|x-y|} d y \\
& \quad=4 \pi \begin{cases}\frac{1}{\left|x-x_{0}\right|} \int_{0}^{\left|x-x_{0}\right|} \rho^{2} g(\rho) d \rho+\int_{\left|x-x_{0}\right|}^{R} \rho g(\rho) d \rho & \text { if }\left|x-x_{0}\right| \leqq R, \\
\frac{1}{\left|x-x_{0}\right|} \int_{0}^{R} \rho^{2} g(\rho) d \rho & \text { if }\left|x-x_{0}\right|>R .\end{cases}
\end{aligned}
$$

Integration by parts in (4.2) leads to the identity

$$
\begin{aligned}
& \int_{B\left(x_{0}, R\right)} g\left(\left|x_{0}-y\right|\right) p(y, t) d y \\
& =\int_{B\left(x_{0}, R\right)}(v(y, t) \otimes v(y, t)): \nabla_{y}^{2}\left(\frac{1}{\left|y-x_{0}\right|} \int_{0}^{\left|y-x_{0}\right|} \rho^{2} g(\rho) d \rho\right. \\
& \left.+\int_{\left|y-x_{0}\right|}^{R} \rho(\rho) d \rho\right) \\
& +\int_{0}^{R} \rho^{2} g(\rho) d \rho \quad(v(y, t) \otimes v(y, t)): \nabla_{y}^{2}\left(\frac{1}{\left|y-x_{0}\right|}\right) d y .
\end{aligned}
$$

Taking $g(\rho)=1 / \rho$ and then $g(\rho)=1$, we arrive at identities (4.1).

Arguing by contradiction, let us denote by $t_{0}$ the first moment of time when singular points of $v$ appear. It is known that $t_{0}>0$ and, for any $T$ in the range $0<T<t_{0}$, our solution $v$ is smooth on $\left.\mathbb{R}^{3} \times\right] 0, T$ ] (see [15]). In particular, for any domain $\Omega \subset \mathbb{R}^{3}$ and for any $0<\delta<t_{0}$, the function $v$ together with the associated pressure $p$ forms a suitable weak solution to the Navier-Stokes equations in the space-time cylinder $\left.Q_{\delta, t_{0}} \equiv \Omega \times\right] \delta, t_{0}\left[\right.$. Moreover, for any $0 \leqq t<t_{0}$, the 
following two identities hold:

$$
\int_{\mathbb{R}^{3}}|v(x, t)|^{2} d x+2 \int_{0}^{t} \int_{\mathbb{R}^{3}}\left|\nabla v\left(x, t^{\prime}\right)\right|^{2} d x d t^{\prime}=\int_{\mathbb{R}^{3}}\left|v_{0}(x)\right|^{2} d x
$$

and

$$
\begin{aligned}
& \int_{\mathbb{R}^{3}}|v(x, t)|^{2} \phi(x) d x+2 \int_{0}^{t} \int_{\mathbb{R}^{3}}\left|\nabla v\left(x, t^{\prime}\right)\right|^{2} \phi(x) d x d t^{\prime} \\
& =\int_{\mathbb{R}^{3}}\left|u_{0}(x)\right|^{2} \phi(x) d x+\int_{0}^{t} \int_{\mathbb{R}^{3}}\left|v\left(x, t^{\prime}\right)\right|^{2} \Delta \phi(x) d x d t^{\prime} \\
& \quad+\int_{0}^{t} \int_{\mathbb{R}^{3}}\left(\left|v\left(x, t^{\prime}\right)\right|^{2}+2 p\left(x, t^{\prime}\right)\right) v\left(x, t^{\prime}\right) \cdot \nabla \phi(x) d x d t^{\prime}
\end{aligned}
$$

for any $\phi \in C_{0}^{\infty}\left(\mathbb{R}^{3}\right)$. They imply

$$
\begin{aligned}
\int_{\mathbb{R}^{3}}|v(x, t)|^{2}(1-\phi(x)) d x+2 \int_{0}^{t} \int_{\mathbb{R}^{3}}\left|\nabla v\left(x, t^{\prime}\right)\right|^{2}(1-\phi(x)) d x d t^{\prime} \\
=\int_{\mathbb{R}^{3}}\left|u_{0}(x)\right|^{2}(1-\phi(x)) d x-\int_{0}^{t} \int_{\mathbb{R}^{3}}\left|v\left(x, t^{\prime}\right)\right|^{2} \Delta \phi(x) d x d t^{\prime} \\
\quad-\int_{0}^{t} \int_{\mathbb{R}^{3}}\left(\left|v\left(x, t^{\prime}\right)\right|^{2}+2 p\left(x, t^{\prime}\right)\right) v\left(x, t^{\prime}\right) \cdot \nabla \phi(x) d x d t^{\prime}
\end{aligned}
$$

for any $\phi \in C_{0}^{\infty}\left(\mathbb{R}^{3}\right)$ and for all $0 \leqq t<t_{0}$. We note that, by the multiplicative inequality, we have

$$
\|u\|_{3, Q_{t_{0}}}^{3} \leqq c_{1} t_{0}^{\frac{1}{4}}\|u\|_{2, \infty, Q_{t_{0}}}^{\frac{3}{2}}\|\nabla u\|_{2, Q_{t_{0}}}^{\frac{3}{2}} \leqq c_{1} t_{0}^{\frac{1}{4}}\left\|u_{0}\right\|_{2, Q_{t_{0}}}^{3}
$$

where $\left.Q_{t_{0}}=\mathbb{R}^{3} \times\right] 0, t_{0}\left[\right.$ and $c_{1}$ is an absolute constant. Dividing (4.1) by $\frac{4 \pi}{3} R^{2}$ and taking the limit as $R \rightarrow 0+0$, we obtain

$$
3 p(x, t)+|u(x, t)|^{2}=\frac{3}{4 \pi} \int_{\mathbb{R}^{3}} \nabla_{y}^{2}\left(\frac{1}{|y-x|}\right):(v(y, t) \otimes v(y, t)) d y .
$$

The theory of singular integrals and (4.4) tell us that

$$
\|p\|_{\frac{3}{2}, Q_{t_{0}}}<+\infty
$$


Thus, by an appropriate choice of the cut-off function $\phi$, we find from (4.3)-(4.5) that

$$
\lim _{R \rightarrow+\infty} \sup _{0 \leqq t<t_{0}} \int_{\mathbb{R}^{3}-B(0, R)}|u(x, t)|^{2} d x=0 .
$$

Finally, since

$$
\liminf _{t \rightarrow t_{0}-0} \int_{\mathbb{R}^{3}-B(0, R)}|u(x, t)|^{2} d x \geqq \int_{\mathbb{R}^{3}-B(0, R)}\left|u\left(x, t_{0}\right)\right|^{2} d x,
$$

we have

$$
\lim _{R \rightarrow+\infty} \sup _{0 \leqq t \leqq t_{0}} \int_{\mathbb{R}^{3}-B(0, R)}|u(x, t)|^{2} d x=0 .
$$

Assume first that condition (2.9) holds. Then, (4.1) can be transformed to the form

$$
\begin{aligned}
& -\frac{1}{2 R} \int_{B\left(x_{0}, R\right)}|v(x, t)|^{2} d x+\frac{3}{2 R} \int_{B\left(x_{0}, R\right)}\left(|v(x, t)|^{2}+2 p(x, t)\right) d x \\
& =\int_{B\left(x_{0}, R\right)} \frac{1}{\left|x-x_{0}\right|}\left(|v(x, t)|^{2}+2 p(x, t)\right) d x-\int_{B\left(x_{0}, R\right)} \frac{1}{\left|x-x_{0}\right|}\left|\widetilde{v}^{x_{0}}(x, t)\right|^{2} d x \\
& =R^{2} \int_{\mathbb{R}^{3}-B\left(x_{0}, R\right)} K\left(x, x_{0}\right):(v(x, t) \otimes v(x, t)) d x
\end{aligned}
$$

where

$$
K\left(x, x_{0}\right) \equiv \nabla_{x}^{2}\left(\frac{1}{\left|x-x_{0}\right|}\right) .
$$

From (4.7), it follows that

$$
\begin{aligned}
& \frac{1}{2 R} \int_{B\left(x_{0}, R\right)}|v(x, t)|^{2} d x \\
& =\frac{3}{2 R} \int_{B\left(x_{0}, R\right)}\left(|v(x, t)|^{2}+2 p(x, t)\right) d x \\
& \quad+\int_{B\left(x_{0}, R\right)} \frac{1}{\left|x-x_{0}\right|}\left|\widetilde{v}^{x_{0}}(x, t)\right|^{2} d x \\
& \quad-\int_{B\left(x_{0}, R\right)} \frac{1}{\left|x-x_{0}\right|}\left(|v(x, t)|^{2}+2 p(x, t)\right) d x \\
& \leqq
\end{aligned}
$$




$$
\begin{aligned}
& +\int_{B\left(x_{0}, R\right)} \frac{1}{\left|x-x_{0}\right|}\left|\widetilde{v}^{x_{0}}(x, t)\right|^{2} d x \\
& +\int_{B\left(x_{0}, R\right)} \frac{1}{\left|x-x_{0}\right|}\left[g(x, t)-\left(|v(x, t)|^{2}+2 p(x, t)\right)\right] d x
\end{aligned}
$$

and thus

$$
\begin{aligned}
& \frac{1}{2 R} \int_{B\left(x_{0}, R\right)}|v(x, t)|^{2} d x \\
& \leqq \\
& \quad \frac{1}{2} \int_{B\left(x_{0}, R\right)} \frac{1}{\left|x-x_{0}\right|} g(x, t) d x \\
& \quad+\int_{B\left(x_{0}, R\right)} \frac{1}{\left|x-x_{0}\right|}\left|\widetilde{v}^{x_{0}}(x, t)\right|^{2} \\
& \quad+\int_{B\left(x_{0}, R\right)} \frac{1}{\left|x-x_{0}\right|}\left[g(x, t)-\left(|v(x, t)|^{2}+2 p(x, t)\right)\right] d x .
\end{aligned}
$$

In addition, we are going to use the identity

$$
\begin{aligned}
\frac{1}{2} \int_{B\left(x_{0}, R\right)} & \frac{1}{\left|x-x_{0}\right|} g(x, t) d x+\int_{B\left(x_{0}, R\right)} \frac{1}{\left|x-x_{0}\right|}\left|\widetilde{v}^{x_{0}}(x, t)\right|^{2} \\
& +\int_{B\left(x_{0}, R\right)} \frac{1}{\left|x-x_{0}\right|}\left[g(x, t)-\left(|v(x, t)|^{2}+2 p(x, t)\right)\right] d x \\
= & \frac{3}{2} \int_{B\left(x_{0}, R\right)} \frac{1}{\left|x-x_{0}\right|} g(x, t) d x-R^{2} \\
& \times \int_{\mathbb{R}^{3}-B\left(x_{0}, R\right)} K\left(x, x_{0}\right):(v(x, t) \otimes v(x, t)) d x .
\end{aligned}
$$

According to (2.7), we can show from (4.8) and (4.9) that, for any $x_{0} \in \mathbb{R}^{3}$ and for any $\left.R \in] 0, R_{0}\left(t_{0}\right)\right]$, the following bound is valid:

$$
\begin{aligned}
& \frac{1}{2 R} \int_{B\left(x_{0}, R\right)}\left|v\left(x, t_{0}\right)\right|^{2} d x \\
& \leq \frac{1}{2} \int_{B\left(x_{0}, R\right)} \frac{1}{\left|x-x_{0}\right|} g\left(x, t_{0}\right) d x \\
& \quad+\int_{B\left(x_{0}, R\right)} \frac{1}{\left|x-x_{0}\right|}\left|\tilde{v}^{x_{0}}\left(x, t_{0}\right)\right|^{2}
\end{aligned}
$$




$$
\begin{aligned}
& +\int_{B\left(x_{0}, R\right)} \frac{1}{\left|x-x_{0}\right|}\left[g\left(x, t_{0}\right)-\left(\left|v\left(x, t_{0}\right)\right|^{2}+2 p\left(x, t_{0}\right)\right)\right] d x \\
\leqq & \frac{1}{2} \int_{B\left(x_{0}, R_{0}\right)} \frac{1}{\left|x-x_{0}\right|} g\left(x, t_{0}\right) d x+\int_{B\left(x_{0}, R_{0}\right)} \frac{1}{\left|x-x_{0}\right|}\left|\widetilde{v}^{x_{0}}\left(x, t_{0}\right)\right|^{2} \\
& +\int_{B\left(x_{0}, R_{0}\right)} \frac{1}{\left|x-x_{0}\right|}\left[g\left(x, t_{0}\right)-\left(\left|v\left(x, t_{0}\right)\right|^{2}+2 p\left(x, t_{0}\right)\right)\right] d x \\
= & \frac{3}{2} \int_{B\left(x_{0}, R_{0}\right)} \frac{1}{\left|x-x_{0}\right|} g\left(x, t_{0}\right) d x \\
& -R_{0}^{2} \int_{\mathbb{R}^{3}-B\left(x_{0}, R_{0}\right)} K\left(x, x_{0}\right):\left(v\left(x, t_{0}\right) \otimes v\left(x, t_{0}\right)\right) d x \\
\leqq & \frac{3}{2} A\left(t_{0}\right)+\frac{c_{2}}{R_{0}\left(t_{0}\right)}\left\|v\left(\cdot, t_{0}\right)\right\|_{2, \mathbb{R}^{3}}^{2},
\end{aligned}
$$

where $c_{2}$ is an absolute constant. This is one of the crucial points of our argument. Together with Lemma 3.2, it implies that the function $t \mapsto u(\cdot, t)$ is continuous from the left at the point $t_{0}$ as a function with values in $L_{2}\left(\mathbb{R}^{3} ; \mathbb{R}^{3}\right)$. To see this, we notice that Lemma 3.2 gives

$$
\lim _{t \rightarrow t_{0}-0} \int_{B(0, r)}\left|v(x, t)-v\left(x, t_{0}\right)\right|^{2} d x=0
$$

for any $r>0$. But then (4.6) yields

$$
\lim _{t \rightarrow t_{0}-0} \int_{\mathbb{R}^{3}}\left|v(x, t)-v\left(x, t_{0}\right)\right|^{2} d x=0 .
$$

Let $\varepsilon_{\star}=\varepsilon_{\star}(1)$ be the number of Lemma 3.3. Fix an arbitrary $x_{0}$ in $\mathbb{R}^{3}$. There exists a positive number $R_{\star} \leqq R_{0}\left(t_{0}\right)$ such that

$$
\begin{aligned}
\frac{\varepsilon_{\star}}{2}> & \frac{1}{2} \int_{B\left(x_{0}, R_{\star}\right)} \frac{1}{\left|x-x_{0}\right|} g\left(x, t_{0}\right) d x+\int_{B\left(x_{0}, R_{\star}\right)} \frac{1}{\left|x-x_{0}\right|}\left|\widetilde{v}^{x_{0}}\left(x, t_{0}\right)\right|^{2} d x \\
& +\int_{B\left(x_{0}, R_{\star}\right)} \frac{1}{\left|x-x_{0}\right|}\left[g\left(x, t_{0}\right)-\left(\left|v\left(x, t_{0}\right)\right|^{2}+2 p\left(x, t_{0}\right)\right)\right] d x \\
= & \frac{3}{2} \int_{B\left(x_{0}, R_{\star}\right)} \frac{1}{\left|x-x_{0}\right|} g\left(x, t_{0}\right) d x \\
& -R_{\star}^{2} \int_{\mathbb{R}^{3}-B\left(x_{0}, R_{\star}\right)} K\left(x, x_{0}\right):\left(v\left(x, t_{0}\right) \otimes v\left(x, t_{0}\right)\right) d x .
\end{aligned}
$$


But, by the continuity condition (2.8) and by (4.10), the function

$$
\begin{aligned}
t \mapsto & \frac{3}{2} \int_{B\left(x_{0}, R_{\star}\right)} \frac{1}{\left|x-x_{0}\right|} g(x, t) d x \\
& -R_{\star}^{2} \int_{\mathbb{R}^{3}-B\left(x_{0}, R_{\star}\right)} K\left(x, x_{0}\right):(v(x, t) \otimes v(x, t)) d x
\end{aligned}
$$

is continuous from the left at the point $t_{0}$. Therefore there exists a positive number $\delta_{\star} \leqq \sqrt{t_{0} / 2}$ such that

$$
\begin{aligned}
\frac{\varepsilon_{\star}}{2}> & \frac{1}{2} \int_{B\left(x_{0}, R_{\star}\right)} \frac{1}{\left|x-x_{0}\right|} g(x, t) d x+\int_{B\left(x_{0}, R_{\star}\right)} \frac{1}{\left|x-x_{0}\right|}\left|\widetilde{v}^{x_{0}}(x, t)\right|^{2} \\
& +\int_{B\left(x_{0}, R_{\star}\right)} \frac{1}{\left|x-x_{0}\right|}\left[g(x, t)-\left(|v(x, t)|^{2}+2 p(x, t)\right)\right] d x
\end{aligned}
$$

for all $t \in\left[t_{0}-\delta_{\star}^{2}, t_{0}\right]$. Then (4.8) leads to the estimate

$$
\begin{aligned}
\frac{1}{2 R} & \int_{B\left(x_{0}, R\right)}|v(x, t)|^{2} d x \\
\leq & \frac{1}{2} \int_{B\left(x_{0}, R\right)} \frac{1}{\left|x-x_{0}\right|} g(x, t) d x \\
& +\int_{B\left(x_{0}, R\right)} \frac{1}{\left|x-x_{0}\right|}\left|\widetilde{v}^{x_{0}}(x, t)\right|^{2} \\
& +\int_{B\left(x_{0}, R\right)} \frac{1}{\left|x-x_{0}\right|}\left[g(x, t)-\left(|v(x, t)|^{2}+2 p(x, t)\right)\right] d x \\
\leq & \frac{1}{2} \int_{B\left(x_{0}, R_{\star}\right)} \frac{1}{\left|x-x_{0}\right|} g(x, t) d x+\int_{B\left(x_{0}, R_{\star}\right)} \frac{1}{\left|x-x_{0}\right|}\left|\widetilde{v}^{x_{0}}(x, t)\right|^{2} \\
& +\int_{B\left(x_{0}, R_{\star}\right)} \frac{1}{\left|x-x_{0}\right|}\left[g(x, t)-\left(|v(x, t)|^{2}+2 p(x, t)\right)\right] d x \\
< & \frac{\varepsilon_{\star}}{2}{ }
\end{aligned}
$$

being valid for all $\left.R \in] 0, R_{\star}\right]$ and for all $t \in\left[t_{0}-\delta_{\star}^{2}, t_{0}\right]$. The last bound and Lemma 3.3 imply that $z_{0}=\left(x_{0}, t_{0}\right)$ is a regular point. Since $x_{0}$ was chosen arbitrarily, the function $u$ is Hölder continuous at any point of the set $\mathbb{R}^{3} \times\left[t_{0} / 2, t_{0}\right]$ and, therefore, $\nabla u \in C\left(\left[t_{0} / 2, t_{0}\right] ; L_{2}\left(\mathbb{R}^{3} ; \mathbb{M}^{3}\right)\right)$. In turn, this implies the existence of a number $t_{1}>t_{0}$ with the property that $\nabla u \in C\left(\left[t_{0}, t_{1}\right] ; L_{2}\left(\mathbb{R}^{3} ; \mathbb{M}^{3}\right)\right)$. So, one can state that $\nabla u \in L_{\infty}\left(0, t_{1} ; L_{2}\left(\mathbb{R}^{3} ; \mathbb{M}^{3}\right)\right)$. Therefore, $u$ is regular in some neighborhood of any point $\left(x, t_{0}\right), x \in \mathbb{R}^{3}$. But this contradicts the definition of $t_{0}$. 
Assume now that conditions (2.10) holds. This case is treated more or less in the same way as the previous one. In particular, it follows from (4.1) that

$$
\begin{aligned}
& \frac{1}{R} \int_{B\left(x_{0}, R\right)}\left(|v(x, t)|^{2}+3(p(x, t)+g(x, t))\right) d x \\
& =\frac{3}{R} \int_{B\left(x_{0}, R\right)} g(x, t) d x-2 \int_{B\left(x_{0}, R\right)} \frac{1}{\left|x-x_{0}\right|} g(x, t) d x \\
& \quad+\int_{B\left(x_{0}, R\right)} \frac{1}{\left|x-x_{0}\right|}\left(\left|\widehat{v}^{x_{0}}(x, t)\right|^{2}+2(p(x, t)+g(x, t))\right) d x \\
& \leqq \\
& \quad \int_{B\left(x_{0}, R\right)} \frac{1}{\left|x-x_{0}\right|} g(x, t) d x \\
& \quad+\int_{B\left(x_{0}, R\right)} \frac{1}{\left|x-x_{0}\right|}\left(\left|\widehat{v}^{x_{0}}(x, t)\right|^{2}+2(p(x, t)+g(x, t))\right) d x
\end{aligned}
$$

and

$$
\begin{aligned}
\int_{B\left(x_{0}, R\right)} & \frac{1}{\left|x-x_{0}\right|} g(x, t) d x \\
& +\int_{B\left(x_{0}, R\right)} \frac{1}{\left|x-x_{0}\right|}\left(\left|\widehat{v}^{x_{0}}(x, t)\right|^{2}+2(p(x, t)+g(x, t))\right) d x \\
= & 3 \int_{B\left(x_{0}, R\right)} \frac{1}{\left|x-x_{0}\right|} g(x, t) d x \\
& +R^{2} \int_{\mathbb{R}^{3}-B\left(x_{0}, R\right)} K\left(x, x_{0}\right):(v(x, t) \otimes v(x, t)) d x .
\end{aligned}
$$

Next, by (2.7), (4.11), and (4.12), we can show that, for any $x_{0} \in \mathbb{R}^{3}$ and for any $\left.R \in] 0, R_{0}\left(t_{0}\right)\right]$, the following bound is valid:

$$
\frac{1}{R} \int_{B\left(x_{0}, R\right)}\left|v\left(x, t_{0}\right)\right|^{2} d x \leqq 3 A\left(t_{0}\right)+\frac{c_{2}}{R_{0}\left(t_{0}\right)}\left\|v\left(\cdot, t_{0}\right)\right\|_{2, \mathbb{R}^{3}}^{2} .
$$

This estimate, Lemma 3.2, and (4.6) imply (4.10). 
Let $\varepsilon_{\star}=\varepsilon_{\star}(1)$ be the number of Lemma 3.3. Fix an arbitrary $x_{0}$ in $\mathbb{R}^{3}$. There exists a positive number $R_{\star} \leqq R_{0}\left(t_{0}\right)$ such that

$$
\begin{aligned}
\frac{\varepsilon_{\star}}{2}> & \int_{B\left(x_{0}, R_{\star}\right)} \frac{1}{\left|x-x_{0}\right|} g\left(x, t_{0}\right) d x \\
& +\int_{B\left(x_{0}, R_{\star}\right)} \frac{1}{\left|x-x_{0}\right|}\left(\left|\widehat{v}^{x_{0}}\left(x, t_{0}\right)\right|^{2}+2\left(p\left(x, t_{0}\right)+g\left(x, t_{0}\right)\right)\right) d x \\
= & 3 \int_{B\left(x_{0}, R_{\star}\right)} \frac{1}{\left|x-x_{0}\right|} g\left(x, t_{0}\right) d x \\
& +R_{\star}^{2} \int_{\mathbb{R}^{3}-B\left(x_{0}, R_{\star}\right)} K\left(x, x_{0}\right):\left(v\left(x, t_{0}\right) \otimes v\left(x, t_{0}\right)\right) d x .
\end{aligned}
$$

By continuity condition (2.8) and by (4.10)-(4.13), the function

$$
\begin{aligned}
& t \mapsto 3 \int_{B\left(x_{0}, R_{\star}\right)} \frac{1}{\left|x-x_{0}\right|} g(x, t) d x \\
& \quad+R_{\star}^{2} \int_{\mathbb{R}^{3}-B\left(x_{0}, R_{\star}\right)} K\left(x, x_{0}\right):(v(x, t) \otimes v(x, t)) d x
\end{aligned}
$$

is continuous from the left at the point $t_{0}$. Therefore there exists a positive number $\delta_{\star} \leqq \sqrt{t_{0} / 2}$ such that

$$
\begin{aligned}
& \frac{1}{R} \int_{B\left(x_{0}, R\right)}|v(x, t)|^{2} d x \\
& \quad \leqq \\
& \quad \int_{B\left(x_{0}, R\right)} \frac{1}{\left|x-x_{0}\right|} g(x, t) d x \\
& \quad+\int_{B\left(x_{0}, R\right)} \frac{1}{\left|x-x_{0}\right|}\left(\left|\widehat{v}^{x_{0}}(x, t)\right|^{2}+2(p(x, t)+g(x, t))\right) d x \\
& \leqq \\
& \quad \int_{B\left(x_{0}, R_{\star}\right)} \frac{1}{\left|x-x_{0}\right|} g(x, t) d x \\
& \quad+\int_{B\left(x_{0}, R_{\star}\right)} \frac{1}{\left|x-x_{0}\right|}\left(\left|\widehat{v}^{x_{0}}(x, t)\right|^{2}+2(p(x, t)+g(x, t))\right) d x \\
& <\frac{\varepsilon_{\star}}{2}
\end{aligned}
$$

for all $\left.R \in] 0, R_{\star}\right]$ and for all $t \in\left[t_{0}-\delta_{\star}^{2}, t_{0}\right]$. And, again, this estimate together with Lemma 3.3 leads to the same contradiction with the definition of $t_{0}$. Theorem 2.2 is proved. 
Acknowledgements. G.S. wishes to acknowledge the hospitality of the School of Mathematics at the University of Minnesota, where a part of this work was carried out. The work of V.S. was supported in part by NSF grant DMS 9877055.

\section{References}

1. Beirao da Veiga, H., A Sufficient Condition on the Pressure for the Regularity of Weak Solutions to the Navier-Stokes Equations, J. Math. Fluid Mech. 2 (2000), 99-106.

2. Berselli, L. C., Sufficient conditions for regularity of the Solution of the Navier-Stokes equations, Math. Meth. Appl. Sci. 22 (1999), 1079-1085.

3. Berselli, L. C. \& Galdi, G.-P., Regularity criterion involving the pressure for weak solutions to the Navier-Stokes equations, Dipartimento di Matematica Applicata, Universita di Pisa, Preprint No. 2001/10.

4. Caffarelli, L., Kohn, R.-V. \& Nirenberg, L., Partial regularity of suitable weak solutions of the Navier-Stokes equations, Comm. Pure Appl. Math. 35 (1982), 771-831.

5. Chae, D. \& Lee, H., Regularity criterion in terms of pressure for the Navier-Stokes equations, Institute for Mathematics and its Applications, University of Minnesota, Preprint No. 1614, 1999.

6. Constantin, P. \& Fefferman, Ch., Direction of vorticity and problem of global regularity for the Navier-Stokes equations, Indiana Univ. Math. J. 42 (1993), 775-789.

7. FreHSE, J. \& RŮŽIČKA, M., Regularity for the stationary Navier-Stokes equations in bounded domains, Arch. Rational Mech. Anal. 128 (1994), 361-380.

8. Giga, Y., Solutions for semilinear parabolic equations in $L^{p}$ and regularity of weak solutions of the Navier-Stokes equations, J. Diff. Equations 62 (1986), 186-212.

9. Giga, Y. \& SoHr, H., Abstract $L^{p}$-estimates for the Cauchy problem with applications to the Navier-Stokes equations in exterior domains, J. Funct. Anal. 102 (1991), 72-94.

10. Hopf, E., Über die Anfangswertaufgabe für die hydrodynamischen Grundgleichungen, Math. Nachrichten, 4 (1950-51), 213-231.

11. Ladyzhenskaya, O. A., Mathematical problems of the dynamics of viscous incompressible fluids, Fizmatgiz, Moscow 1961; English translation, Gordon and Breach, New York-London, 1969.

12. LadyzhensKaya, O. A., On uniqueness and smoothness of generalized solutions to the Navier-Stokes equations, Zapiski Nauchn. Seminar POMI 5 (1967), 169-185.

13. Ladyzhenskaya, O. A., Mathematical problems of the dynamics of viscous incompressible fluids, 2nd edition, Nauka, Moscow 1970.

14. Ladyzhenskaya, O. A. \& Seregin, G. A., On partial regularity of suitable weak solutions to the three-dimensional Navier-Stokes equations, J. Math. Fluid Mech. 1 (1999), 356-387.

15. Leray, J., Sur le mouvement d'un liquide visqueux emplissant l'espace, Acta Math. 63 (1934), 193-248.

16. Lin, F.-H., A new proof of the Caffarelly-Kohn-Nirenberg theorem, Comm. Pure Appl. Math. 51 (1998), 241-257.

17. Kaniel, S. \& Shinbrot, M., Smoothness of weak solutions of the Navier-Stokes equations, Arch. Rational Mech. Anal. 24 (1967), 302-324.

18. Klainerman, S., PDE as a unified subject. GAFA 2000 (Tel Aviv, 1999). Geom. Funct. Anal. 2000, Special Volume, Part I, 279-315.

19. Maremonti, P. \& Solonnikov, V. A., On the estimate of solutions of evolution Stokes problem in anisotropic Sobolev spaces with a mixed norm, Zap. Nauchn. Sem. LOMI 223 (1994), 124-150.

20. NeČas, J. \& Neústupa, J., New condition for local regularity of a suitable weak solution to the Navier-Stokes equation, Preprint.

21. Scheffer, V., Hausdorff measure and the Navier-Stokes equations, Commun. Math. Phys. 55 (1977), 97-112. 
22. Serrin, J., On the interior regularity of weak solutions of the Navier-Stokes equations, Arch. Rational Mech. Anal. 9 (1962), 187-195.

23. SERrin, J., The initial value problem for the Navier-Stokes equations. Nonlinear Problems (R. LANGER ed.), pp. 69-98, Madison: The University of Wisconsin press, 1963.

24. Solonnikov, V. A., Estimates of solutions to the linearized system of the Navier-Stokes equations, Trudy of the Steklov Math. Inst. 70 (1964), 213-317.

25. Struwe, M., On partial regularity results for the Navier-Stokes equations, Comm. Pure Appl. Math. 41 (1988), 437-458.

26. Struwe, M., Regular solutions of the stationary Navier-Stokes equations on $\mathbb{R}^{5}$, Math. Ann. 302 (1995), 719-741.

Steklov Institute of Mathematics at St.Petersburg St.Peterburg, Russia

and

School of Mathematics, University of Minnesota

Minneapolis, USA

(Accepted January 25, 2002)

Published online May 3, 2002 - (C) Springer-Verlag (2002) 